\title{
$\Omega$
}

Patrycja Rzepka-Wrona

[1]

Uniwersyteckie Centrum Kliniczne im prof. Kornela Gibińskiego Śląskiego

Uniwersytetu Medycznego, Katowice, Poland.

\section{My night on call in Poland}

I am a young doctor who graduated from the Medical University of Silesia in 2014. After 13 months of postgraduate training, passing a state examination and successful completion of the admission process I made a decision to specialise in Respiratory Medicine. I started working in a large clinical hospital that provides junior doctors with excellent opportunities for training and career development. The respiratory unit is highly specialised and deals with sleep-related medical problems, interstitial lung diseases and respiratory failure, which may be treated using noninvasive mechanical ventilation. Along with my training in respiratory medicine I decided to start working as an emergency room doctor in a different hospital, in order to improve my skills in treating lifethreatening conditions.

My on-calls start at $15: 30 \mathrm{~h}$ and finish at $07: 30 \mathrm{~h}$ in the working week and if I work on weekends, these are $24 \mathrm{~h}$ long shifts. If a patient requires a multidisciplinary approach, it is possible to do a hand-over and transfer them to a more specialised facility or ask for a medical consultation. It is also possible to contact a local pharmacology and toxicology department in order to obtain information on how to treat drug or alcohol poisoning. I cover the emergency department; however, when I decide that a patient should be admitted to the hospital I am also obliged to design a long-term therapeutic plan, including imaging and laboratory tests to be performed over the next few days. The local hospital where I work as an emergency room doctor does not have a respiratory ward, so respiratory patients are hospitalised in an internal medicine ward, where it is possible to use a respirator for intubated patients. Noninvasive mechanical ventilation techniques are available in more specialised units, like clinical hospitals which have respiratory wards. In the respiratory ward, it is possible to initiate continuous positive airway pressure (CPAP) treatment in patients with acute or chronic respiratory failure, the majority of whom are people suffering from chronic obstructive pulmonary disease (COPD).

When in doubt, I may call an older, more experienced doctor who covers the internal medicine and geriatric ward and who is a specialist in internal medicine. We both sleep in the hospital overnight. Generally, I see $10-15$ patients during an on call, however, there may be up to 20 or more on weekends. The situation varies, as in colder months there are definitely more patients with COPD exacerbations. If there are public festivals nearby I have to deal with toxic substance poisoning, such as alcohol intoxication resulting in respiratory depression.

Since I started working on call in the emergency room in the local hospital, I have realised that dyspnoea or shortness of breath are possibly the most frequent complaints you hear, no matter the time of day.

Pulmonary oedema is one of the most frequent maladies in patients suffering from uncompensated cardiac failure. Usually these people are delivered to the emergency room by a medical first-aid service team. These patients also manifest with escalating dyspnoea and are unable to speak a proper sentence without making pauses to inhale rapidly. In Polish hospitals, an infusion pump with furosemide is often administered. If a patient has hypertension or tachypnoea, nitroglycerine and morphine are also available. Traditionally, morphine is administered subcutaneously and intravenously at the same time in small doses.

If CPAP was available in the emergency room, this method would probably become increasingly popular in such cases. However, such equipment is mostly available in respiratory wards or intensive care units.

What I did not expect was for a patient with developing pulmonary oedema to come to the emergency room alone and, moreover, to say that her main concern was unstable blood pressure values. I measured her blood pressure twice, and
Cite as: Rzepka-Wrona P. My night on call in Poland. Breathe 2016; 12: 291-292. 
it was $\sim 120 / 70 \mathrm{mmHg}$. I asked the patient specifically what she meant by unstable blood pressure values. She said that she had measured it repeatedly that night, and her systolic blood pressure had ranged from 115 to $125 \mathrm{mmHg}$, which made her really concerned about myocardial infarction or cerebral stroke risk. I assured her that these values stay within the normal range; however, she seemed unimpressed and told me that such differences in results obtained at 5-10 min time intervals appeared extremely dangerous to her and such a condition required immediate medical intervention. In spite of clearly visible respiratory effort, cyanosis and loud auscultatory phenomena she vigorously denied feeling dyspnoeic, even though she was unable to express herself without making numerous pauses to take a deep breath.

Another case I was quite impressed by was an elder lady delivered to the emergency room by her daughter. The patient was distressed and claimed her condition appeared quite suddenly a few hours earlier, making her fear for her life. She stated that she always felt quite healthy otherwise and had never suffered from a serious disease. After she removed her sweater and shawl, I could see that her face, neck and arms were flushed and swollen. There were also hard masses palpable within her neck and supraclavicular area. On computed tomography there were multiple tumours, most probably enlarged lymph nodes, which constricted the trachea and superior caval vein. Due to increasing dyspnoea the patient was sedated and intubated and, unfortunately, died 3 h later.

I am convinced that working as an emergency room doctor, although extremely stressful and demanding at times, will be rewarding in terms of gaining confidence and experience as a doctor. Having the opportunity to work with my friend, a more experienced doctor and a very friendly and understanding person at the same time, makes me feel grateful. Hopefully, this experience will add some value to my future performance as a respiratory specialist.

\section{Conflict of interest}

None declared. 